\title{
Clinical and neurophysiological assessment of immunoglobulin therapy in five patients with multifocal motor neuropathy
}

\author{
Giancarlo Comi, Stefano Amadio, Giuseppe Galardi, Raffaella Fazio, Raffaello Nemni
}

\begin{abstract}
High dose intravenous immunoglobulin (IVIg) is an effective treatment for demyelinating neuropathies. IVIg was given to five patients with multifocal motor neuropathy, a motor neuropathy showing a clinical syndrome of asymmetrical weakness and amyotrophy, electrophysiological evidence of motor conduction block and, in many cases, high titres of serum anti-GM1 antibodies. Muscle strength was evaluated by a conventional score before and after each IVIg course. In all patients there was relevant improvement on muscle strength after each immunoglobulin course, but in most cases the clinical benefits partially declined after three to eight weeks. At the eight month follow up, however, the pretreatment examination showed a significant improvement compared with the initial evaluation. The effects of each IVIg course were still present after a number of courses. Electrophysiological study revealed a decrease in conduction block in one or more nerves in all patients. However, conduction block was unchanged or increased in other sites. IVIg treatment did not affect anti-GM1 antibody titres.
\end{abstract}

Multifocal motor neuropathy (MMN) is a recently recognised neurological disorder, characterised by asymmetrical weakness and muscle wasting, fasciculations and cramps, usually predominant in the upper limbs. ${ }^{1}$ The diagnosis is based on nerve conduction studies revealing the presence of multifocal conduction block, confined to the motor axons. Pathological findings of chronic demyelination have been found at sites of conduction block. ${ }^{2}$ High titres of autoantibodies against GM1 and others nerve glycolipids are frequently found in the serum of $M M N$ patients, suggesting an immunemediated pathogenesis. ${ }^{3}$

Based on these findings, several immunomodulating therapeutic approaches have been proposed. Treatment with steroids and plasma-exchange failed to show clinical benefits in patients with MMN. Cyclophosphamide has proved to be an effective therapy in the majority of the patients treated ${ }^{4}$; because of its toxicity, however, cyclophosphamide cannot be considered the first choice treatment especially in patients with mild neurological disability.

Because of previous reports of its efficacy in other possible immune-mediated neuropathies $^{56}$ intravenous infusion of high dose human immunoglobulin (IVIg) has been tested in a few $M M N$ cases. ${ }^{7-9}$ Positive effects have been found in almost all cases. We report our experience with immunoglobulin therapy in five patients with $M M N$.

\section{Patients and methods}

Five consecutive patients with clinical and neurophysiological diagnosis of MMN entered the study. All patients were males, and the mean age was 39.6 years (range 23-52). They showed a clinical syndrome of asymmetrical weakness and muscle atrophy without relevant sensory loss, which lasted for at least six months (table 1). Three patients had been previously treated with steroids, plasma exchange or cyclophosphamide, but the treatment had been considered unsatisfactory. The degree of neurological disability was assessed by means of the Medical Research Council (MRC) scale. Five muscles were examined in each limb and the scores added (maximum score $=100$ ).

Motor conduction of the peroneal, median and ulnar nerves was bilaterally examined in all patients. The peroneal nerve was stimulated at the ankle, below and above the fibular head. The median and ulnar nerves were stimulated at the wrist, elbow, axilla and Erb's point; the motor conduction of the ulnar nerve through the cubital groove was also evaluated, to rule out the more obvious causes of conduction block. A stimulus was considered supramaximal if its intensity was at least threefold of the lower intensity stimulus required for the motor threshold. Additional study of proximal conduction was carried out by means of F-wave. Sensory conduction of the median, ulnar and sural nerves of both sides were also studied. The proximal-to-distal ratio of the compound motor action potential amplitude and area over close nerve segments was also measured. The conduction block (CB) and temporal dispersion (TD) were defined according to the criteria of Lange et al. ${ }^{10}$ Anti-GM1 antibodies titres were measured by enzyme-linked immunosorbent assay (ELISA) according to Sadiq et al. ${ }^{11}$ The upper normal limit in our laboratory is $1: 600$.

All patients received human immunoglobulin (Sandoglobulin Sandoz; Basel, Switzerland) at doses of $0.4 \mathrm{~g} / \mathrm{kg}$ over a period of five days. All patients had at least two IVIg administrations. Clinical assessment was performed at day 1 and 8 of each IVIg 
Table 1 Clinical, laboratory and neurophysiological features

\begin{tabular}{|c|c|c|c|c|c|c|c|c|c|c|}
\hline \multirow[b]{2}{*}{ Patients } & \multicolumn{5}{|c|}{ Clinical and laboratory features } & \multicolumn{5}{|c|}{ Neurophysiological features } \\
\hline & Sex/age & $\begin{array}{l}\text { Illness } \\
\text { duration }\end{array}$ & Course & Distribution & $\begin{array}{l}\text { Anti-GM1 } \\
\text { antibodies }\end{array}$ & $\begin{array}{l}\text { Conduction } \\
\text { block }\end{array}$ & $\begin{array}{l}\text { Temporal } \\
\text { dispersion }\end{array}$ & $\begin{array}{l}\text { Axonal } \\
\text { loss* }\end{array}$ & $N C V$ & $F$-wave \\
\hline 1 & $M / 34$ & 2 years & Stepwise & $\begin{array}{l}\text { Right arm } \\
P=D \\
\text { Left arm } \\
D\end{array}$ & Absent & $\begin{array}{l}\text { R median Erb-ax } \\
R \text { ulnar Erb-ax } \\
R \text { Mc Erb-ax }\end{array}$ & $\begin{array}{l}\text { R median } \\
\text { Erb-ax and ax-El } \\
\text { L median } \\
\text { Erb-ax and El-W } \\
\text { L ulnar } \\
\text { Ax-El and El-W }\end{array}$ & Severe & Normal & Absent \\
\hline 2 & $M / 48$ & 3 years & Progressive & $\begin{array}{l}\text { Left arm } \\
P=D\end{array}$ & Absent & $\begin{array}{l}\text { R ulnar Erb-ax } \\
\text { L median Erb-ax } \\
\text { L ulnar Erb-ax } \\
\text { L Mc Erb-ax }\end{array}$ & L Median Erb-ax & Severe & Normal & Absent \\
\hline 3 & $M / 41$ & 2 years & Progressive & $\begin{array}{l}\text { Upper limbs } \\
\text { D } \\
\text { left leg } \\
\text { D }\end{array}$ & $1: 2,400$ & $\begin{array}{l}\mathrm{R} \text { median } \mathrm{El}-\mathrm{W} \\
\mathrm{L} \text { median } \mathrm{El}-\mathrm{W}\end{array}$ & $\begin{array}{l}\text { R ulnar } \\
\text { Ax-El and El-W } \\
\text { L ulnar El-W } \\
\text { L peroneal FH-An }\end{array}$ & Severe & $\begin{array}{l}\text { Slightly } \\
\text { reduced }\end{array}$ & Abnormal \\
\hline 4 & $M / 23$ & 1 year & Progressive & $\begin{array}{l}\text { Upper limbs } \\
\text { (right }>\text { left) } \\
\text { D }\end{array}$ & $1: 20,000$ & $\begin{array}{l}\text { R median Erb-ax } \\
\mathbf{R} \text { ulnar ax-El } \\
\text { L median Erb-ax } \\
\text { L ulnar Erb-ax }\end{array}$ & - & Severe & Normal & Absent \\
\hline 5 & $M / 52$ & 12 years & Progressive & $\begin{array}{l}\text { Upper limbs } \\
\text { (right > left) } \\
\text { D }\end{array}$ & $1: 80,000$ & $\begin{array}{l}\text { L median Erb-ax } \\
\mathbf{R} \text { ulnar Erb-ax }\end{array}$ & $\begin{array}{l}\text { L median El-W } \\
\text { L ulnar Erb-ax }\end{array}$ & Severe & $\begin{array}{l}\text { Slightly } \\
\text { reduced }\end{array}$ & Abnormal \\
\hline
\end{tabular}

^Evaluated with both electrophysiological (cMAP amplitude) and electromyographic (fibrillation potentials, positive sharp waves and fasciculations; motor unit action potentials increased in duration and amplitude; decreased recruitment at maximum voluntary effort) criteria.

NCV: nerve conduction velocity; P = proximal; D = distal; Erb: Erb's point; Ax: axilla; El: elbow; W: wrist; FH: fibular head; An: ankle.

course; a long term evaluation of neurological disability was carried out at the eighth month.

Electrophysiological evaluation was performed in all cases before each IVIg course. In two cases neurophysiological investigation was also performed at the end of the first IVIg course.

\section{Results}

CLINICAL ASSESSMENT

After the first IVIg course all patients noticed an improvement of strength. In all cases the MRC Disability Score confirmed an increase in muscle power (table 2). The improvement was fast, lasted between three to eight weeks, before there was a progressive decline. The level of disability of the pretreatment examination was, however, never reached. At the eight month follow up, there was an improvement in all patients on the MRC score compared with the pretreatment values. Mean (SD) values of the MRC score increased after therapy: the differences between the average score on day 8 and day 1 after either the first or second IVIg course $\left[\begin{array}{lllll}85.0 & (6.0) & v & 91.0\end{array}\right.$ $(5.6) ; 86.6(5.9) \quad v 92.4(4.3)$ respectively] was statistically significant $(p<0.001)$. At the eight month follow up the mean (SD) MRC disability score showed a significant increase compared with the mean basal value [from $85.0(6)$ to $93.4(2 \cdot 6) ; \mathrm{p}<0.05$ ].

ELECTROPHYSIOLOGICAL TESTS

Electrophysiological tests provided evidence of conduction block in two or more motor nerves in all patients. The neurophysiological changes after IVIg treatment are shown in table 2. A complete resolution of motor conduction block was not observed. A reduction of conduction block in one or more nerves was found in all patients. When compared with the overall number for conduction block and temporal dispersion, the cMAP ratio increased to at least $50 \%$ in $21 \%(5 / 24)$ of nerve segments with conduction block, after both the first and second IVIg course; minor improvement ( $>20 \%$ ) was found in $12.5 \%$ of the cases, again after both the first and the second IVIg course.

Table 2 Clinical and neurophysiological follow up

\begin{tabular}{|c|c|c|c|c|c|c|c|c|c|}
\hline \multirow[b]{3}{*}{ Patients } & \multicolumn{5}{|c|}{ MRC Disability Score } & \multicolumn{4}{|c|}{ Conduction block/temporal dispersion improvement ${ }^{*}$} \\
\hline & \multicolumn{2}{|c|}{ 1st IVIg trial } & \multicolumn{2}{|c|}{ 2nd IVIg trial } & \multirow[b]{2}{*}{ 8th month } & \multicolumn{2}{|l|}{ After 1st IVIg trial } & \multicolumn{2}{|l|}{ After 2nd IVIg trial } \\
\hline & Before & After & Before & After & & $>50 \% *$ & $>20 \% *$ & $>50 \%{ }^{\circ}$ & $>20 \%{ }^{\circ}$ \\
\hline 1 & 84 & 92 & 86 & 91 & 90 & R ulnar ax-El & R median ax-El & $\begin{array}{l}\text { R median Erb-ax } \\
R \text { Mc Erb-ax }\end{array}$ & L ulnar ax-El \\
\hline 2 & 90 & 97 & 91 & 97 & 91 & $\begin{array}{l}\text { L median Erb-ax } \\
\text { L ulnar Erb-ax } \\
\text { L Mc Erb-ax }\end{array}$ & - & - & - \\
\hline 3 & 91 & 95 & 94 & 97 & 97 & R median El-W & L ulnar El-W & R median El-W & $\begin{array}{l}\text { R ulnar } \\
\text { Ax-El and El-W }\end{array}$ \\
\hline 4 & 76 & 83 & 80 & 88 & 93 & - & L median Erb-ax & $\mathbf{R}$ median Erb-ax & - \\
\hline 5 & 84 & 88 & 82 & 89 & 95 & - & - & L ulnar Erb-ax & - \\
\hline
\end{tabular}

^Percentage of improvement of proximal-to-distal cMAP amplitude and area ratio as compared to pre-treatment values. - : No significant changes. 
No significant changes on the mean distal cMAP amplitude of the nerves tested were found after the single treatment or at the eight month examination. The amount of fibrillation and fasciculation potentials was unmodified after the treatment.

\section{IMMUNOLOGICAL FEATURES}

In three patients titres of anti-GM1 antibodies higher than the normal range were found. No significant, treatment-induced changes on anti-GM1 antibodies titres were observed.

\section{Discussion}

The main result of the present IVIg trial is the substantial, but short lived, clinical benefit experienced by all patients. In the affected limbs, improvement was sometimes dramatic. In patients 1 and 2 who had a conduction block on the musculocutaneous nerve (making the elbow virtually impossible to flex) normal strength was restored after the first few days of IVIg infusion. Benefit to the small hand muscles, with some improvement in handwriting, was also observed in patient 3 with prevalent involvement of the median or ulnar nerve. As the effect was mostly transient, all patients had at least a second course of IVIg therapy. Retreatment produced an improvement in all patients similar to that observed after the first IVIg course. The amount and duration of clinical effects were highly variable from patient to patient. At the eight month follow up, at least one month after the last IVIg course, all patients showed a significant clinical benefit, indicating some long lasting effect of the treatment, in spite of fluctuations related to each IVIg course.

Our data seem therefore to confirm the results of previously published open studies suggesting that IVIg therapy could be the treatment of choice in short term management of MMN. ${ }^{7}$ Chaudhry et $a l^{7}$ reported improvement in strength in nine patients with MMN, lasting for an average of two months; IVIg courses were repeated in six patients, each time with favourable results. The authors, however, did not report data about long term residual neurological deficit. Nobile-Orazio et $a l^{8}$ followed five patients with $\mathrm{MMN}$ for six to 12 months; four improved and in one patient recovery was complete and continued for 12 months without any additional treatment. In three cases, clinical benefit lasted 20 to 30 days.

Though the effects of the IVIg therapy on electrophysiological tests has previously been emphasised, we have not observed a complete resolution of the conduction block or an increase of distal summed cMAP amplitude as reported by others. ${ }^{78}$ We found a substantial increase of the proximal-to-distal cMAP amplitude in a majority of patients, but the CB was persistent. Conversely, no more than $33.5 \%$ of the nerves with a CB or TD showed a significant increase of cMAP ratio. Our findings agree with those of Kaji et al, ${ }^{9}$ who observed little improvement in conduction block after IVIg therapy. This was attributed to either an improvement of myelin function or the resynchronisation of nerve conduction, leading to less phase cancellation. Moreover, we found in a few nerves an extension of CB to additional nerve segments or a deterioration of pre-existing conduction block during the same follow up period. The amount of active denervation at EMG examination was unchanged after therapy. These observations suggest that pathophysiological mechanisms leading to chronic demyelination and secondary axonal loss are not completely blocked during IVIg treatment.

The financial support of Telethon-Italy to the projects 251 and 422 is gratefully acknowledged.

1 Parry GJ, Sumner AJ. Multifocal motor neuropathy. Neurol Clin 1992;10:671-84.

2 Kaji R, Oka N, Tsuji T, Mezaki T, Nishio T, Akiguchi I, Kimura J. Pathological findings at the site of conduction block in multifocal motor neuropathy. Ann Neurol 1993;
33:152-8.

3 Pestronk A. Invited review: motor neuropathies, motor neuron disorders, and antiglycolipid antibodies. Muscle Nerve 1991;14:927-36.

4 Feldman EL, Bromberg MB, Albers JW, Pestronk A. Immunosuppressive treatment in multifocal motor neuropathy. Ann Neurol 1991;30:397-401.

5 Van Der Meche FGA, Schmitz PIM, and the Dutch Guillain-Barré study group. A randomized trial comparing intravenous immune globulin and plasma exchange in Guillain-Barré syndrome. $N$ Eng $\mathcal{f}$ Med 1992;326: $1123-9$.

6 Vermeulen M, Van Doorn PA, Brand A, Strengers PFW, Jennekens FGI, Busch HFM. Intravenous immunoglobulin treatment in patients with chronic inflammatory controlled study. $\mathcal{F}$ Neurol Neurosurg Psychiatry 1993: 56:36-9.

7 Chaudhry V, Corse AM, Cornblath DR, Kuncl RW, Drachman DB, Freimer ML, Miller RG, Griffin JW. Multifocal motor neuropathy: response to human immune globulin. Ann Neurol 1993;33:237-42.

8 Nobile-Orazio E, Meucci N, Barbieri S, Carpo M, Scarlato G. High-dose intravenous immunoglobulin therapy in multifocal motor neuropathy. Neurology 1993;43:537-44.

9 Kaji R, Shibasaki H, Kimura J. Multifocal demyelinating motor neuropathy: cranial nerve involvement and immunoglobulin therapy. Neurology 1992;42:506-9.

10 Lange DJ, Trjaborg W, Latov N, et al. Multifocal motor neuropathy with conduction block: is it a distinct clini-

11 Sadiq SA, Thomas FP, Kilidireas K, et al. The spectrum of neurological disease associated with anti-GM1 antibodies. Neurology 1990;40:1067-72. 